\section{Diálogos docentes para el diseño de un programa de formación en ciudadanía y convivencia para niños, niñas $y$ adolescentes de una Institución Educativa en Colombia}

Teacher's dialogues for the design of a formation program in citizenship and connivance for children and teenagers of an Educational Institution in Colombia

Federico Jose Jimenez Varon* Andres Francisco Olivar Rojas ${ }^{* *}$ Flor Maria Morantes Valencia***

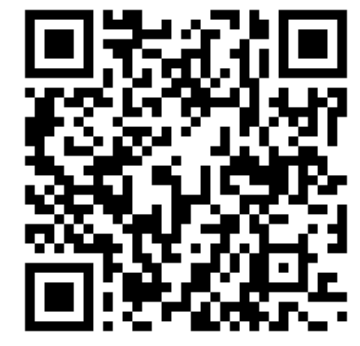

\section{Resumen}

El trabajo que se presenta acá corresponde a las etapas de diseño, planeación y priorización de un proyecto de investigación orientado a la intervención de una problemática de convivencia y clima escolar en una Institución Educativa pública de un municipio de Colombia. Así, el objetivo de este artículo es dar cuenta de un proceso de construcción colectiva del concepto de ciudadanía como objeto pedagógico, así como de una posible estrategia para su enseñanza. Los resultados obtenidos muestran que la reflexión sobre el concepto de ciudadanía implica su operacionalización en una gama de nociones que se solapan y se complementan, dentro de las cuales se encuentran las de familia, escuela y

Filósofo. Magíster en Paz, Desarrollo y Ciudadanía. Corporación Universitaria Minuto de Dios-UNIMINUTO Girardot, Colombia federico.jimenez@uniminuto.edu https://orcid.org/0000-0002-2917-9222

** Comunicador Social y Periodista. Magíster en Estudios Políticos Corporación Universitaria Minuto de DiosUNIMINUTO Girardot, Colombia aolivar@uniminuto.edu https://orcid.org/0000-0003-3504-0347

${ }^{* * *}$ Comunicadora Social y Periodista. Magíster en Antropología Corporación Universitaria Minuto de DiosUNIMINUTO Girardot, Colombia flor.morantes@uniminuto.edu https://orcid.org/0000-0001-7074-6535

\author{
Sinergias educativas \\ Enero - Marzo Vol. 6 - 12021 \\ http://sinergiaseducativas.mx/index.php/revista/ \\ eISSN: 2661-6661 \\ revistasinergia@soyuo.mx \\ Pag 15-29 \\ Recibido: 03 de abril 2020 \\ Aprobado: 19 de noviembre 2020
}


comunidad, derechos humanos, medio ambiente y sexualidad y género. En relación con las técnicas para la implementación de una posible estrategia de enseñanza-aprendizaje, los docentes identificaron al grafiti, la cartografía social, el teatro y la música como elementos lúdicopedagógicos facilitadores del proceso. Estos resultados sugieren la importancia de la recuperación de este tipo de espacios como elementos constitutivos de las realidades educativas, así como la necesidad de un análisis de los imaginarios sociales presentes al interior de esas realidades. La metodología fue cualitativa con un enfoque participativo que combinó técnicas como las entrevistas a profundidad, los grupos focales y los talleres lúdicos. La población constó de 43 profesores y profesoras, que se distribuyeron, para los talleres, en 5 mesas de trabajo en las que diligenció una matriz para la priorización de temáticas, la selección de metodologías y recursos.

Palabras clave: ciudadanía, convivencia, participación, pedagogía, deserción escolar.

\begin{abstract}
The work presented here corresponds to the design, planning and prioritization stages of a research project aimed at the intervention of a problem of connivance and school climate in a public educational institution in a municipality of Colombia. Thus, the objective of this article is to account for a process of collective construction of the concept of citizenship as a pedagogical object, as well as a possible strategy for its teaching. The results show that such reflection on the concept of citizenship implies its operationalization in a range of notions that overlap and complement each other, among which are those of family, school and community, human rights, environment and sexuality-gender. In relation to the techniques for the implementation of a possible teaching-learning strategy, the teachers identified graffiti, social cartography, theater and music as playful-pedagogical elements that facilitate the process. These results suggest the importance of the recovery of this type of spaces as constitutive elements of educational realities, as well as the need for an analysis of the social imaginary present within these realities. The methodology was qualitative with a participatory approach that combined techniques such as in-depth interviews, focus groups and playful workshops. The participants were a group conformed by 43 teachers, that for the workshops was divided in 5 work tables in which they filled out a matrix for the prioritization of topics and the selection of methodologies and resources.
\end{abstract}

Key words: citizenship, connivance, participation, pedagogy, school dropout. 


\section{Introducción}

En el marco del proceso de articulación entre la Corporación Universitaria Minuto de Dios y la Secretaría de Educación Municipal de Girardot, Cundinamarca (SEM) y en virtud de algunos hallazgos obtenidos a parir de un proyecto de investigación sobre los factores de riesgo de la deserción escolar en el municipio (Jiménez Varón, Federico José, \& Rivera Cumbe, Magnolia, 2019), se han identificado una serie de necesidades relacionadas con la convivencia y la formación ciudadana al interior de las instituciones educativas, que se manifiestan en las diferentes formas de violencia (física, verbal, psicológica, etc.) que tienen lugar en las aulas y demás espacios escolares, y que repercuten en las diferentes dimensiones del desarrollo individual de los niños, las niñas y los adolescentes (NNA). La principal consecuencia es la reproducción y perpetuación, en las escuelas y colegios, de los ciclos de violencia presentes en los imaginarios sociales a los que, según el Ministerio de Educación Nacional (2017), recurren los estudiantes como la principal forma de resolver conflictos y diferencias.

El punto de partida consistió entonces en empezar a abrir canales de comunicación con las personas involucradas para explorar los contenidos de esos imaginarios, describirlos y entenderlos en clave dialógica para concertar las acciones que permitan atender el problema desde sus diferentes dimensiones. Fue así como gracias a la gestión y articulación de esfuerzos entre las coordinaciones de Docencia, Investigación y Proyección social de Uniminuto-Centro Regional Girardot, se escogió un equipo interdisciplinar para comenzar un acercamiento con la comunidad escolar de educación básica y media de la ciudad, con el fin de proponer la elaboración de una estrategia de intervención de la problemática señalada, desde el enfoque de la comunicación-educación. El propósito era diseñar un programa de formación que se sustente en estrategias pedagógicas y didácticas provenientes de campos como la lúdica artística y la alfabetización digital, ya que lo que se buscaba era mostrar, tanto a docentes como a los NNA, una alternativa de aprendizaje paralela a la inscrita en los diseños curriculares de la educación tradicional. 
Reconociendo las dinámicas de la opresión de la escuela tradicional en el terreno

Se empezó pues con una serie de reuniones exploratorias con directivos docentes y rectores para detectar el interés que podía suscitar la propuesta en los líderes de procesos. La respuesta favorable fue automática en la mayoría de los casos. Sin embargo, llamó la atención la fuerte resistencia de algunos coordinadores académicos aduciendo razones como la inconveniencia operativa pues no se podía alterar el plan de trabajo de los docentes- y "la falta de novedad de la estrategia", ya que la convivencia y la ciudadanía son temas que, por lineamientos del Ministerio de Educación Nacional de Colombia, deben ser incluidos en los contenidos curriculares de las asignaturas de manera transversal.

Al mismo tiempo, al interior del equipo de profesoras y profesores de Uniminuto, se incubó la idea de que, al provenir de una universidad, lo más natural para los y las docentes de los colegios era pensar que la propuesta que ofrecíamos fuera principalmente académica, con lo que quedaban claras dos cosas: que este era un prejuicio al que nos teníamos que enfrentar, y que al notarlo estábamos seguros de estar tocando las teclas correctas.

En este contexto, los diálogos entre docentes se desarrollaron dentro de una temporalidad difícil de acordar y de planear. Los tiempos de los trabajadores de la educación pública siempre son ajenos a sus voluntades y el hecho de que prime lo urgente sobre lo importante se ha vuelto cosa normal en este tipo de cotidianidades laborales. Cada vez más las actividades administrativas y operativas consumen el tiempo que profesores y profesoras debieran separar para la necesaria reflexión sobre su práctica. La información tampoco llegaba oportunamente a quienes debía llegar, y al principio siempre quedaba la sensación de que el arribo del equipo investigador a los colegios era entendido por los profesores de las instituciones educativas más como el resultado de una adenda de último momento en un orden del día planeado para otros menesteres, que el producto de una planeación. Se entendió luego que la presencia de Uniminuto en esos espacios era justificada por rectores y coordinadores académicos como parte de unas agendas programáticas orientadas al cumplimiento de unos planes maestros del ente regulador, la 
Secretaría de Educación Municipal. Era obvio que las dinámicas de poder estaban mediando y permeando los primeros acercamientos, lo que motivó que el interés se centrara en ese aspecto del discurso semiótico social que no se dice, pero que significa algo.

Sin embargo comenzaron las conversaciones y la disposición favorable hacia la iniciativa siempre estuvo presente, pero oculta detrás de los semblantes propios de quienes son sometidos constantemente a escrutinio en forma de evaluaciones de desempeño y mediciones de calidad, cosas que quizá poco tengan que ver con las luchas diarias de estos docentes que prestan su servicio a una de las poblaciones más atravesadas por lo que desde los paradigmas desarrollistas llaman "condiciones de vulnerabilidad" (Sachs, 1996) y que Huergo (2000) caracteriza como condiciones de precariedad e insignificancia de los espacios sociales. Esos espacios no son físicos sino afectivos (pues sucede que el colegio estaba relativamente bien provisto de bienes y servicios). Poco tiempo pasó para que quedara claro, a partir de las narraciones de los maestros y de las maestras, que muchos de los y las estudiantes consideraban ese como un espacio de afecto más que de formación al que, empero, llegaban los problemas de afuera y se materializaban en expresiones de violencias físicas y simbólicas que son, según las conversaciones, insuficientemente atendidas por los adultos de los respectivos entornos familiares.

Establecido de este modo que el tema de conversación era el problema de convivencia escolar, se llevaron a cabo una serie de mesas de trabajo conformadas por profesores y profesoras de la universidad y de la institución educativa seleccionada para diseñar un plan de formación sobre unos temas que se entendieron como constitutivos del concepto de ciudadanía. Se estableció que, si la ciudadanía era el objeto de formación, entonces esta debía ser entendida como un objeto inacabado, pero siempre a punto de concretarse y que, aunque puede llegar a materializarse un día, puede desvanecerse al día siguiente en ese campo de lucha por el significado que según Huergo (2002) es la cultura.

\section{Materiales y métodos}

La investigación se enmarcó dentro de una metodología cualitativa con enfoque interpretativo basado en la investigación-participativa, 
que buscaba la comprensión del fenómeno social desde las distintas realidades subjetivas e intersubjetivas que componen la vida cotidiana en contextos específicos (Sandoval, 2002). El diseño metodológico de la propuesta se fundamentó en el rol activo y participativo de la comunidad sujeto, tomando en cuenta su diversidad cultural (Rappaport, 1977 citado en Montero, 2007), así como el desarrollo de sus fortalezas y capacidades, lo que implica la producción de saberes, el fortalecimiento de las capacidades sociales y la activación de procesos de cambio (Saranson, 1974 citado en Montero, 2007).

Se desarrollaron 4 grupos focales, 8 entrevistas individuales semiestructuradas, 5 mesas de trabajo y 2 talleres de retroalimentación con 43 profesores y profesoras de la Institución educativa seleccionada.

Tabla 1. Obejtivos de la muestra

Lugar y fecha I.E Fundadores Ramón Bueno y José Triana de la ciudad de Girardot, Cundinamarca-Colombia -marzo de 2019

Propósito de Aplicación de la matriz de priorización de temáticas, selección de metodologías y la actividad recursos.

Objetivos

- Clasificar numéricamente en orden de importancia las temáticas propuestas para los talleres y las actividades pedagógicas a realizar.

- Identificar las estrategias pedagógicas que se emplearán para la posterior ejecución de los talleres.

- Articular los talleres y actividades pedagógicas con las diferentes áreas de formación existentes en la I.E.

\section{Resultados}

En primera instancia se debe partir de la necesidad, tanto conceptual como práctica, de entender la noción de ciudadanía más allá de su dimensión electoral-representativa, que es la que ha primado históricamente y es, a su vez, la que ha despojado de la dimensión política y cultural a la noción de ciudadanía. De igual forma, se debe partir de entender lo político, no solo como lo estatal, sino como cualquier acción, individual o colectiva -pero principalmente 
colectiva- tendiente a modificar un orden de cosas. Esta concepción de lo político es útil para la discusión teórica, pues se verá cómo las prácticas ciudadanas se profundizan cuando asumimos que todos, independientemente de nuestra condición socioeconómica, somos actores políticos capaces de modificar nuestro entorno. En este orden de ideas, se tratará de operacionalizar el concepto de ciudadanía mediante categorías de análisis como territorio, participación ciudadana, diversidad sexual, derechos humanos y democracia radical.

Así, el territorio no es únicamente un lugar físico-espacial. Es, también, una construcción social, histórica y cultural en la que se ponen en juego significados y sentidos para un grupo humano (Saque, 2016). Esto implica que en el territorio se construyen relaciones, afectos, encuentros y desencuentros entre individuos con intereses disímiles. En este espacio, tanto físico como simbólico, los agentes comunican sus sentires. Dicha comunicación, o puesta en común de símbolos, es el primer paso para hablar de participación ciudadana.

La participación ciudadana en Colombia siempre se ha entendido desde dimensiones instrumentales referidas, primordialmente, a mecanismos de participación ciudadana consagrados en la Constitución Política de 1991. Así, el voto, el referendo, el plebiscito, el cabildo abierto, entre otros, son estrategias estatales para fomentar la injerencia directa de los ciudadanos en los asuntos públicos. Sin embargo, esto limita la participación a una dimensión puramente jurídico-estatal, pues únicamente se necesita ser mayor de edad y portar la cédula de ciudadanía para acceder a estos mecanismos. Por ello, a tono con la propuesta conceptual aquí planteada, la participación también puede darse desde espacios físicos y simbólicos en los que se expresen sentires respecto de lo público. Así, un niño, niña o adolescente, por ejemplo, puede en el espacio de su colegio manifestar su posición sobre asuntos referidos a su entorno inmediato (de este modo emergen subcategorías de análisis como el uso del espacio público, seguridad-inseguridad, vida saludable, espacios para la práctica de artes y deportes, entre otros aspectos). Así, el concepto y la práctica de la participación ciudadana desbordan los límites de lo jurídico-estatal y en esta medida el fomento de las artes resulta fundamental para crear órdenes 
simbólicos transgresores de relaciones de poder naturalizadas en un núcleo social. ¿Pero de qué forma?

El cuerpo y la sexualidad, por ejemplo, son objetos colonizados por órdenes simbólicos que imponen formas de manifestar tanto la identidad de género como la orientación de género. (Viveros, 2016). El entorno escolar suele ser particularmente cruel con aquellos jóvenes que manifiestan identidades y orientaciones de género que se salen del binarismo de género y la heterosexualidad obligatoria $o$ heteronormativa, valores hegemónicos impuestos por una cultura patriarcal, colonial, etnocéntrica y androcéntrica que niega cualquier orden ajeno a lo socialmente aceptado. De esta forma, la música, la pintura, el canto, el teatro, y cualquier performance -en cualquier formato, analógico o digital-, son herramientas desinstitucionalizadas, des-estatalizadas, para promover la participación ciudadana, pues la manifestación artística puede llegar a ser disruptiva y por ello, puede generar una deconstrucción del orden simbólico que considera "anormal" o "desviado" identidades y orientaciones de género diferentes. Esto conlleva a crear visiones de mundo más respetuosas de la diferencia y por tanto más incluyentes.

La ciudadanía del siglo XXI es una ciudadanía diversa, con múltiples clivajes que implican y exigen la profundización de la democracia y de la ciudadanía en todos los ámbitos de la micropolítica. Las demandas sociales del siglo XX giraban en torno a la lucha entre burguesía y proletariado. Las demandas sociales del siglo XXI, más allá de la lucha de clases, giran ahora en torno a la defensa del territorio, de la diversidad sexual, del medio ambiente, de la democratización del ejercicio de la ciudadanía y de lo que Chantal Mouffe (Canales, 2011) denomina democracia radical, es decir, la posibilidad de otorgar a la ciudadanía una capacidad de agencia que vaya más allá de lo representativo-electoral, para canalizar las demandas por la defensa del territorio, la diversidad sexual, la participación ciudadana y los derechos humanos. 


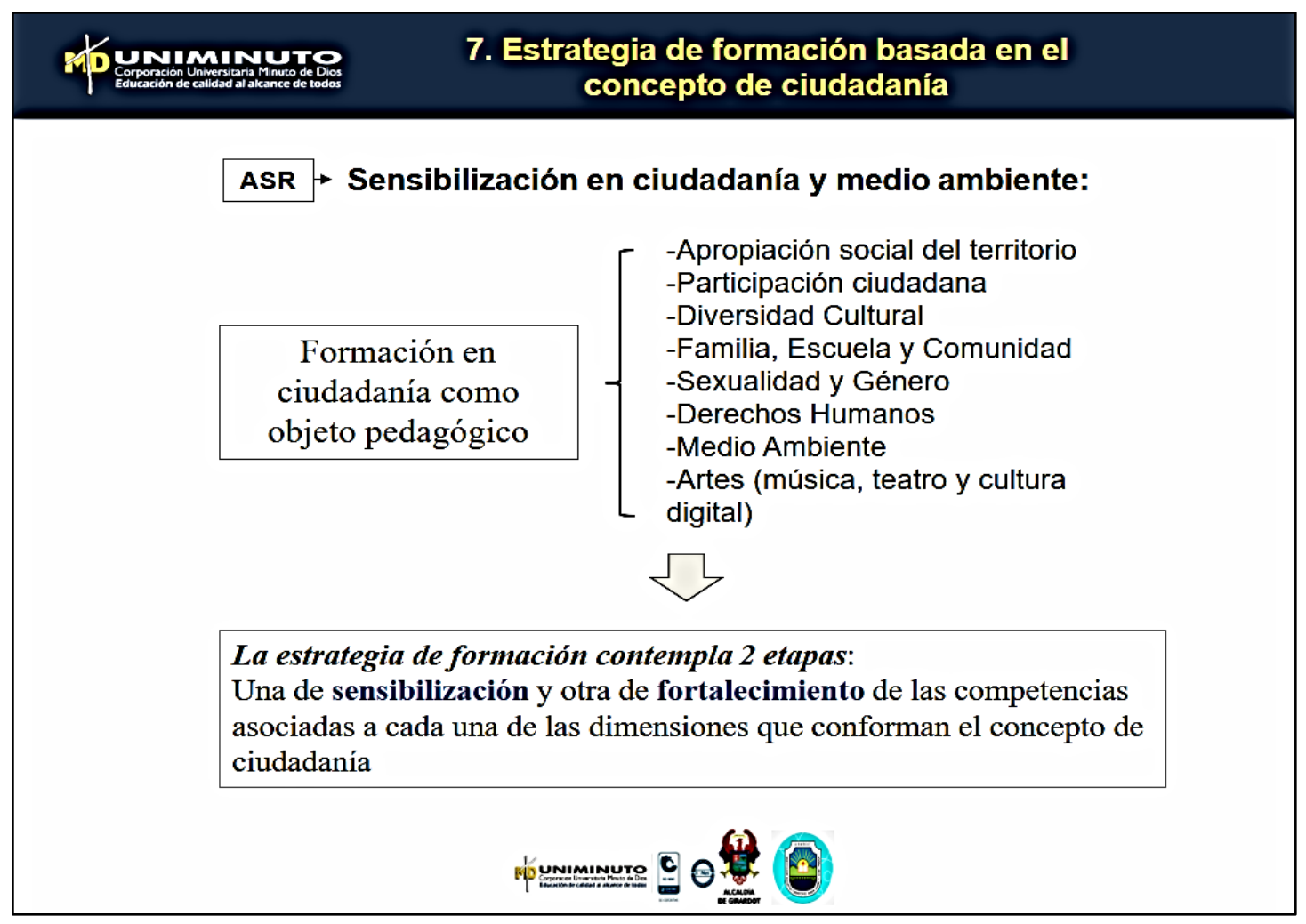

Graficol: Concepto de ciudadanía desglosado a partir de la estrategia de lluvia de ideas.

\section{b. Priorización de temáticas y estrategias lúdico-pedagógicas para la implementación del plan de formación.}

A continuación, en la Tabla 2 "Valoración numérica de las temáticas", se expone de manera detallada la calificación entregada por cada uno de los 5 equipos de trabajo que participaron en la actividad. Es importante señalar que la lectura de la escala inicia en 1 y termina en 7; siendo 1 el tema con mayor prioridad de ejecución (según los docentes participantes) y 7 el tema con menor prioridad de ejecución.

Tabla 2. Valoración numérica de las temáticas

TEMA /VALORACIÓN
NUMÉRICA

$\begin{array}{lllll}\text { APROPIACIÓN SOCIAL } & 1 & 1 & 3 \\ \text { DEL TERRITORIO } & \end{array}$

\section{CIUDADANA}




\begin{tabular}{|c|c|c|c|c|c|c|}
\hline DIVERSIDAD HUMANA & 1 & & 1 & 1 & 1 & 1 \\
\hline $\begin{array}{l}\text { FAMILIA, ESCUELA Y } \\
\text { COMUNIDAD }\end{array}$ & 4 & & 1 & & & \\
\hline DERECHOS HUMANOS & & 2 & 1 & & 2 & \\
\hline MEDIOS AMBIENTE & & 2 & & 1 & & 2 \\
\hline $\begin{array}{l}\text { SEXUALIDAD } \\
\text { GÉNERO }\end{array}$ & & 1 & 2 & 2 & & \\
\hline
\end{tabular}

De otro lado, la aplicación de la matriz también permitió conocer las principales estrategias pedagógicas que emplearían los docentes según la temática a desarrollar. En la siguiente tabla se ilustran los resultados obtenidos:

Tabla 3. Estrategias pedagógicas según la temática

\begin{tabular}{|c|c|c|}
\hline Temática & Estrategia pedagógica & $\begin{array}{l}\text { Puntuación } \\
\text { obtenida }\end{array}$ \\
\hline $\begin{array}{l}\text { Familia, escuela y } \\
\text { comunidad }\end{array}$ & Cartografía social & 4 \\
\hline Derechos humanos & Graffiti & 5 \\
\hline Medio ambiente & Teatro & 3 \\
\hline Sexualidad y género & Cultura digital & 3 \\
\hline Diversidad humana & Música & 3 \\
\hline $\begin{array}{l}\text { Participación } \\
\text { ciudadana }\end{array}$ & Cerámica & 3 \\
\hline $\begin{array}{l}\text { Apropiación social } \\
\text { del territorio }\end{array}$ & Teatro & 4 \\
\hline
\end{tabular}

En línea con lo anterior, en la siguiente tabla se muestran los resultados entregados por los docentes de la IE, frente a la vinculación de asignaturas a cada una de las temáticas propuestas. Se decidió, asimismo, que la metodología será la de la lúdica artística, pues 
se persiguen dos objetivos: i) recuperar para los NNA el espacio vital de la expresión artística a través la lúdica, espacio que paulatinamente ha sido desplazado por el poder central del currículo de la educación bancaria; y ii) explorar los universos simbólicos expresados por los niños y las niñas, a través de la lectura de las imágenes y los objetos producidos en los espacios.

Tabla 4. Asignaturas vinculadas a cada temática

Temática

Familia, escuela y comunidad

Derechos humanos

Medio ambiente

Ciencias sociales e informática

Sexualidad y género

Lenguaje, artística y educación física

Diversidad humana

Artística, español y ciencias sociales

Participación ciudadana

Matemáticas y ciencias naturales

Apropiación social del territorio

\section{Asignaturas vinculadas}

Ciencias sociales, ética y ciencias naturales

Español, inglés y educación física

Ética, religión y ciencias naturales

Por último, se relacionan los recursos y materiales sugeridos por los docentes para el desarrollo de las actividades según la estrategia pedagógica seleccionada.

Tabla 4. Recursos y materiales asociados a cada estrategia pedagógica

\begin{tabular}{llll}
\hline Temática & Estrategia pedagógica & \multicolumn{2}{l}{ Recursos y materiales } \\
\hline $\begin{array}{l}\text { Familia, escuela y } \\
\text { comunidad }\end{array}$ & Cartografía social & $\begin{array}{l}\text { Cartulinas } \\
\text { reciclaje, material de } \\
\text { vinilos } \\
\text { marcadores, }\end{array}$ \\
\hline $\begin{array}{l}\text { Derechos } \\
\text { humanos }\end{array}$ & Graffiti & $\begin{array}{l}\text { Aerosoles, telas, papel } \\
\text { periódico }\end{array}$ & \\
\hline
\end{tabular}




\begin{tabular}{|c|c|c|}
\hline Medio ambiente & Teatro & $\begin{array}{l}\text { Trajes, tallerista profesional, } \\
\text { escenografía, sonido, luces }\end{array}$ \\
\hline $\begin{array}{l}\text { Sexualidad } \\
\text { género }\end{array}$ & Cultura digital & $\begin{array}{l}\text { Video beam, sonido, luces, } \\
\text { internet, computadores, } \\
\text { tablets }\end{array}$ \\
\hline $\begin{array}{l}\text { Diversidad } \\
\text { humana }\end{array}$ & Música & $\begin{array}{l}\text { Sonido, escenografía, trajes, } \\
\text { micrófonos }\end{array}$ \\
\hline $\begin{array}{l}\text { Participación } \\
\text { ciudadana }\end{array}$ & Cerámica & $\begin{array}{l}\text { Tallerista profesional, } \\
\text { arcilla, pinturas pinceles }\end{array}$ \\
\hline $\begin{array}{l}\text { Apropiación } \\
\text { social del } \\
\text { territorio }\end{array}$ & Teatro & $\begin{array}{l}\text { Trajes, tallerista profesional, } \\
\text { escenografía, sonido, luces }\end{array}$ \\
\hline
\end{tabular}

\section{Discusión}

El concepto de ciudadanía fue desglosado y abordado desde lo que se entendió como sus diferentes dimensiones constitutivas, a saber, la apropiación social del territorio, la participación ciudadana, la diversidad, la sexualidad y el género, la triada familia-escuelacomunidad y el medio ambiente. La estrategia de formación diseñada contempla 2 etapas: una de sensibilización y otra de fortalecimiento de las competencias asociadas a cada una de las dimensiones que conforman el concepto de ciudadanía.

Dicha estrategia está orientada a generar en los NNA reflexiones y actitudes sobre el papel que juegan como individuos en las prácticas ciudadanas, a través de actividades lúdico-pedagógicas que los sensibilicen sobre los principales problemas que impiden los cambios necesarios para la construcción de escenarios de convivencia y paz. Estas estrategias y herramientas permiten interrogar e interpretar, desde la observación comprensiva, la realidad a partir de la formación en prospectiva de un nuevo sujeto que se reconoce como ciudadano y agente de cambio.

Siguiendo a Freire, se puede afirmar que el establecimiento del universo temático, es una meta que está aún por lograrse a pesar de 
que se haya establecido y acordado que el diálogo es el punto de partida. En este sentido, se debe hacer énfasis en la necesidad de hacer visible el potencial de estos maestros y maestras como agentes sociales capaces de movilizar procesos culturales, potencial que sería deseable alimentar desde iniciativas que estimulen el intercambio dialógico de experiencias educativas que surgen desde espacios de formación alternativos. El reconocimiento de la ruptura del tejido existente entre la escuela y la comunidad por parte de la mayoría de los actores escolares, sería un primer paso para que ambas voces - la de la comunidad y la de la escuela- sean mutuamente escuchadas.

\section{Referencias}

Canales Urriola, J. (2011). Chantal Mouffe, En torno a lo político, Fondo de Cultura Económica, Buenos Aires, 2007, Polis [En línea]. Recuperado de: http://journals.openedition.org/polis/244

Corporación Universitaria Minuto de Dios - Uniminuto (2014) proyecto educativo institucional del sistema Recuperado: http://www.uniminuto.edu/documents/941377/1434225/Proy ecto+Educativo+Institucional+2013.pdf/849a034b-2ee8448c-9aa9-93e2cef4a317

Freire, P. La Pedagogía del Oprimido. Capítulo III. La esencia de la educación cómo práctica. Ciudad de México, México: Siglo XXI Editores: Recuperado de http://www.servicioskoinonia.org/biblioteca/general/FreirePe dagogiadelOprimido.pdf

Gobierno digital Colombia (2018). ESTADISTICAS EN EDUCACION BASICA POR MUNICIPIO. Recuperado el 31 de enero de 2019 de: https://www.datos.gov.co/Educacin/ESTADISTICAS-EN-EDUCACION-BASICA-PORMUNICIPIO/nudc-7mev

Gómez Díaz, R. (2010). La alfabetización visual: retos para un mundo que aspira a la interculturalidad. En R. Gómez Díaz, \& M. C. Agustín Lacruz, (eds.) Polisemias visuales. Aproximaciones a la alfabetización visual en la sociedad intercultural. Salamanca: Universidad de Salamanca. 
Recuperado de http://eprints.rclis.org/15914/1/978-84-7800166-8-0021-0037.pdf

Huergo, J. (2000). Comunicación / educación Itinerarios transversales. Universidad de la Plata- Argentina. Recuperado de:

http://www.sedguaviare.gov.co/attachments/426_Comunicaci\%C3 \%B3n\%20Huergo\%20(1).pdf

Jiménez Varón, F. (2018). Alfabetización visual como medio para la comprensión de la interculturalidad en Girardot. Perspectivas, (8), 28-33. Recuperado a partir de https://revistas.uniminuto.edu/index.php/Pers/article/view/16 07

Jiménez Varón, Federico José, \& Rivera Cumbe, Magnolia. (2019). Obstáculos metodológicos en el estudio de la deserción escolar del Sistema Público Educativo de Girardot. Conrado, 15(68), 149-153. Epub 02 de septiembre de 2019. Recuperado en 17 de junio de 2020, de http://scielo.sld.cu/scielo.php?script=sci_arttext\&pid=S199086442019000300149\&lng=es\&tlng=es.

Ministerio de Educación Nacional-MEN (2018). Estadísticas sectoriales datos abiertos. Recuperado de: https://www.mineducacion.gov.co/1759/w3-propertyvalue57271.html

Marti, J. (2018). La investigación - acción participativa. Estructura y fases. En: redcimas.org. Recuperado de: http://www.redcimas.org/wordpress/wpcontent/uploads/2012/ 08/m_JMarti_IAPFASES.pdf

Montero, M (2007), Introducción a la psicología comunitaria, desarrollo, conceptos y procesos, Paidós, Buenos Aires.

Sandoval, Carlos. (2002). Investigación cualitativa. Recuperado de: https://panel.inkuba.com/sites/2/archivos/manual\%20colombi $\mathrm{a} \% 20$ cualitativo.pdf

Saquet, M. (2016). Territorios Rurales entre crisis y perspectivas de desarrollo territorial con autonomía: La agricultura campesina 
(agro)ecológica. Eutopía, Revista De Desarrollo Económico

Territorial, 10, 57-76. Recuperado de:

https://revistas.flacsoandes.edu.ec/eutopia/article/view/2410/1522

Viveros, M (2016). La interseccionalidad: una aproximación situada a la dominación. Debate feminista 52 (1-7) Recuperado de http://www.debatefeminista.cieg.unam.mx/wpcontent/uploads/2016/12/articulos/052_completo.pdf

W. SACHS (editor), Diccionario del desarrollo. Una guía del conocimiento como poder, PRATEC, Perú, 1996 (primera edición en inglés en 1992), 399 pp. 